\title{
SARS-Cov-2 Infection and Cardiology: Beware of Myocarditis
}

\author{
Marco Antônio Machado Schlindwein ${ }^{1}$, Letícia Caroline Breis ${ }^{1^{*}}$, Isabelle Pastor Bandeira ${ }^{1}$, Rafael M \\ Ronsoni, MD, MsC' ${ }^{1}$, Clizenaldo Torres, $\mathrm{MD}^{2}$ and Marcus Vinicius Magno Gonçalves, MD, PhD
}

${ }^{1}$ Department of Medicine, University of the Region of Joinville, Brazil

${ }^{2}$ Cardiologist, Instituto Neurovie, Santa Catarina, Brazil

*Corresponding author: Letícia Caroline Breis, Student of University of the Region of Joinville, Rua Ministro Calógeras, 439, Bucarein, Joinville, Santa Catarina, 892020-207, Brazil, Tel: 55+47-9-9176-0101

\begin{abstract}
The emergence of SARS-CoV-2 is a challenge in the actual medical scenario. Besides the classical lung and respiratory disease, patients infected with the virus can present with cardiac injury, and pathogenic mechanisms point to direct infection of the heart. In this paper, we seek to show the correlation between cardiology and SARS-CoV-2 infection, especially with regard to myocarditis, which has been shown to be one of the complications in COVID-19.
\end{abstract}

\section{Keywords}

SARS-COV-2, Covid-19, Myocarditis

\section{Background}

SARS-CoV-2 is the virus responsible for coronavirus disease 2019 (COVID-19). The SARS-CoV-2 outbreak initiated in Wuhan, China, [1] is a novel kind of coronavirus with similarities when compared to its proteomics with SARS-CoV [2]. Its main way to get access to the cells is via the angiotensin-converting enzyme 2 (ACE2) [3]. This virus infection's main clinical features are fever (89.1\%), cough $(72.2 \%)$ and fatigue $(42.5 \%)$, the acute severe respiratory syndrome (ARSD) affects $14.8 \%$ of the patients with $96.6 \%$ presenting with computer tomography (CT) abnormalities [4].

There has been evidence of the cardiovascular system compromise as the pandemic progresses. Firstly because the receptor that the virus uses to get access to the cell is the ACE2 receptor [3] and two major drugs used in cardiology, the ACE inhibitors and angiotensin II type 1 receptor blocker, add possible poor prognosis among those with SARS-CoV-2 infection $[5,6]$, although no evidence of harm or benefit is present in the available data [7].

Secondly, systemic arterial hypertension, which is a prevalent cardiovascular condition [8], was shown to carry a 2.36 odds ratio, in a meta-analysis involving 46248 patients, when comparing the severe patients with the no severe group [9] and cardiovascular disease a 3.42 odds ratio in the same study [9], making cardiovascular conditions the major risk factor for severe disease study until now.

Additionally, as risk factors, cardiovascular complications can appear in the progression of COVID-19 disease. These manifestations are in the form of cardiac injury ( $13 \%$ of patients) [10], and elevated troponin levels, $812 \%$ of patients with COVID-19 have values above the $99^{\text {th }}$ percentile, indicating that cardiac injury may play a role in disease progression [11]. In this paper, we will discuss myocarditis as possible causation of this cardiac injury and the mechanisms involved in cardiac damage caused by SARS-COV-2 infection.

\section{Myocarditis Definition}

Myocarditis is classified on a histological and clinical basis. If the patient has signs of heart injury with no better explanation, signs on the electrocardiograph suggestive of myocardial injury or abnormal heart function measured by an echocardiogram or MRI and is asymptomatic, he may be a patient with subclinical myocarditis. In addition to these findings, the patient may also have a clinical syndrome characteristic of myocarditis (acute heart failure, syncope, chest pain or myocardi- 
al pericarditis). Therefore, an acute myocarditis case is likely. For a definitive diagnosis, an endomyocardial biopsy is required [12].

\section{Pathophysiology}

Viral etiology is a well-defined cause of acute myocarditis $[12,13]$. Commonly coxsackie B viruses, influenza and parvovirus B-19 with the heart been secondarily affected after the virus gain access via respiratory or gastrointestinal tract [13], the damage occurs via inflammatory response and direct induce by the virus [13], a discussed mechanism for the SARS-CoV-2 infection [14].

The SARS-CoV epidemic in 2003 brought evidence that this virus gains access to human hearts and causes cardiac damage [15]. Oudit, et al. showed that $35 \%$ of hearts in an autopsy study of the SARS-COV outbreak, in Canada, were positive for the virus RNA [16]. In this same study, the authors show that the heart infection happens in an ACE 2 dependent manner in a murine model [16]. In those who needed mechanical ventilation, subclinical impairments have also been found on the echocardiography of patients with SARS-CoV [17].

Looking at the pandemic scenario today, recent studies support the hypothesis that the heart is a potential target for SARS-CoV-2. Zou and colleagues showed that $7.5 \%$ of myocardial cells have ACE 2 expression and thus being susceptible to SARS-COV-2 infection [18]. Another study also shows that the heart expresses more ACE 2 than the lungs at the site of the primary infection, even though it has a low expression in cardiomyocytes and pointing to an important expression in pericytes that could play a role in myocardial injury and be a target to SARS-COV-2 in the heart by disturbing the microcirculation [19].

\section{Cardiac Involvement SARS-CoV-2}

Cardiac involvement has the potential to play an important role in SARS-CoV-2 infection. In addition to previous SARS-CoV data showing the heart as a possible target, clinical findings of cardiac injury in infected patients $[10,11]$ also show the relationship of SARS-CoV-2 to having one of its targets in the heart [15-19]. New evidence shows that elevated troponin levels after day 4 of hospitalization are associated with non-surviving patients [20] and cardiogenic shock can play a role in up to $33 \%$ of deaths [21]. Chen, et al. also presented indirect evidence of cardiac involvement with $27.5 \%$ and $10 \%$ of SARS-CoV- 2 patients presenting with elevated $\mathrm{N}$-terminal pro-B-type natriuretic peptide and troponin respectively [22].

Recently, a case of cardiac involvement without the pulmonary component was described on a 53-year-old woman with no previous history of cardiac disease, presenting with two-day severe fatigue denying chest pain, dyspnea, and other symptoms, mentioning just a cough and fever a week early. She had elevated cardiac enzymes, electrocardiograph suggesting ischemia, and regional wall abnormalities on the echocardiogram, with no evidence of acute coronary disease in her angiography. She received the diagnosis of acute SARS-COV-2 myocarditis [14].

Besides, other cases had fulminant myocarditis in patients with SARS-COV-2 infection. The first case presents a male with 63 years with no history of cardiovascular disease, who showed up elevated troponin (11.37 $\mathrm{g} / \mathrm{L})$, sinus tachycardia and low left ventricular ejection fraction (LVEF) on the third day of evolution. After treatment the ejection fraction recovered to $68 \%$ [23].

The second one presented with fulminant myopericarditis associated with cardiac tamponade in a 47-yearold female. Other symptoms were shortness of breath, chest pain, dry cough, hypotension, and tachycardia, serum troponin values also increased, but the echocardiogram showed normal ventricular function with pericardial effusion [24].

Cardiac nuclear magnetic resonance (MRI) plays an important role in the management of COVID \& cardiology cases. Therefore, it is possible to search correctly for the diagnostic criteria of myocarditis, as in the case described by Inciardi, et al. with myocardial edema and late gadolinium improvement [14]. It is preferable to value the clinical diagnosis of these patients since their critical condition is usually a limiting factor for endocardial biopsy $[14,22]$.

Treatment is usually supportive. It includes ventilation, hemodynamic support and the standard approach of the intensive care unit, given that there is insufficient evidence for any other treatment, including antiretroviral drugs or corticosteroids $[14,22]$.

\section{Conclusion}

In conclusion, cardiac injury is common $[10,11]$ in SARS-COV-2 infection. These can present as fulminant myocarditis [21,22], or focal myocarditis [14] and this diagnosis should be remembered during this pandemic even without lung disease. Although more research is needed, cardiac injuries are a marker in patients who don't survive the disease [19] and can be caused by direct infection to the heart. Finally, patients infected or suspected for SARS-COV-2 infection with troponin elevation and/or symptoms pointing to myocarditis or cardiac dysfunction should gain more attention and be submitted for a cardiology consultation.

\section{Acknowledgments}

\section{Financial support}

No financial support.

\section{Material support}

No material support. 


\section{Conflict of interest}

All authors declare that there are no conflicts of interest.

\section{References}

1. Huang C, Wang Y, Li X, Ren L, Zhao J, et al. (2020) Clinical features of patients infected with 2019 novel coronavirus in Wuhan, China. Lancet 395: 497-506.

2. Xu J, Zhao S, Teng T, Abdalla AE, Zhu W, et al. (2020) Systematic comparison of two animal-to-human transmitted human coronaviruses: SARS-CoV-2 and SARS-CoV. Viruses 12: E244.

3. Wan Y, Shang J, Graham R, Baric RS, Li F (2020) Receptor recognition by the novel coronavirus from Wuhan: An analysis based on decade-long structural studies of SARS Coronavirus. J Virol 94: e00127-e00220.

4. Sun P, Qie S, Liu Z, Ren J, Li K, et al. (2020) Clinical characteristics of hospitalized patients with SARS-CoV-2 infection: A single-arm meta-analysis. J Med Virol.

5. Diaz JH (2020) Hypothesis: Angiotensin-converting enzyme inhibitors and angiotensin receptor blockers may increase the risk of severe COVID-19. J Travel Med.

6. Kuster GM, Pfister O, Burkard T, Zhou Q, Twerenbold R, et al. (2020) SARS-CoV2: Should inhibitors of the renin-angiotensin system be withdrawn in patients with COVID-19? Eur Heart J.

7. Danser AHJ, Epstein M, Batlle D (2020) Renin-angiotensin system blockers and the COVID-19 Pandemic: At present there is no evidence to abandon renin-angiotensin system blockers. Hypertension.

8. Elliott WJ (2007) Systemic hypertension. Curr Probl Cardiol 32: 201-259.

9. Yang J, Zheng Y, Gou X, Pu K, Chen Z, et al. (2020) Prevalence of comorbidities in the novel Wuhan coronavirus (COVID-19) infection: A systematic review and meta-analysis. Int J Infect Dis 9712: 30136-3.

10. Rodriguez-Morales AJ, Cardona-Ospina JA, Gutiérrez-Ocampo E, Villamizar-Peña R, Holguin-Rivera Y, et al. Clinical, laboratory and imaging features of COVID-19: A systematic review and meta-analysis. Travel Med Infect Dis 101623.

11. Lippi G, Lavie CJ, Sanchis-Gomar F (2020) Cardiac troponin I in patients with coronavirus disease 2019 (COVID-19): Evidence from a meta-analysis. Prog Cardiovasc Dis.
12. Sagar S, Liu PP, Cooper LT Jr (2012) Myocarditis. Lancet 379: 738-747.

13. Pollack A, Kontorovich AR, Fuster V, Dec GW (2015) Viral myocarditis--diagnosis, treatment options, and current controversies. Nat Rev Cardiol 12: 670-680.

14. Inciardi RM, Lupi L, Zaccone G, Italia L, Raffo M, et al. (2020) Cardiac involvement in a patient with coronavirus disease 2019 (COVID-19). JAMA Cardiol.

15. Gu J, Korteweg C (2007) Pathology and pathogenesis of severe acute respiratory syndrome. Am J Pathol 170: 11361147.

16. Oudit GY, Kassiri Z, Jiang C, Liu PP, Poutanen SM, et al. (2009) SARS-coronavirus modulation of myocardial ACE2 expression and inflammation in patients with SARS. Eur $\mathrm{J}$ Clin Invest 39: 618-625.

17. Li SS, Cheng CW, Fu CL, Chan YH, Lee MP, et al. (2003) Left ventricular performance in patients with severe acute respiratory syndrome: A 30-day echocardiographic follow-up study. Circulation 108: 1798-1803.

18. Zou X, Chen K, Zou J, Han P, Hao J, et al. (2020) Single-cell RNA-seq data analysis on the receptor ACE2 expression reveals the potential risk of different human organs vulnerable to 2019-nCoV infection. Front Med.

19. Chen L, Li X, Chen M, Feng Y, Xiong C (2020) The ACE2 expression in human heart indicates new potential mechanism of heart injury among patients infected with SARSCoV-2. Cardiovascular Research 116: 1097-1100.

20. Zhou F, Yu T, Du R, Fan G, Liu Y, et al. (2020) Clinical course and risk factors for mortality of adult inpatients with COVID-19 in Wuhan, China: A retrospective cohort study. Lancet 395: 1054-1062.

21. Ruan Q, Yang K, Wang W, Jiang L, Song J (2020) Clinical predictors of mortality due to COVID-19 based on an analysis of data of 150 patients from Wuhan, China. Intensive Care Med.

22. Chen C, Zhou Y, Wang DW (2020) SARS-CoV-2: A potential novel etiology of fulminant myocarditis. Herz 45: 230232.

23. Zeng JH, Liu YX, Yuan J, Wang FX, Wu WB, et al. (2020) First case of COVID-19 infection with fulminant myocarditis complication: Case report and insights. Preprints.

24. Hua A, O'Gallagher K, Sado D, Byrne J (2020) Life-threatening cardiac tamponade complicating myopericarditis in COVID-19. Eur Heart J. 